\title{
Second harmonic generation of diode laser radiation in $\mathrm{KNbO} 3$
}

\author{
Niko F. van Hulst, Gerard J.T. Heesink, H. de Leeuw, \\ Bouwe Bolger
}

Niko F. van Hulst, Gerard J.T. Heesink, H. de Leeuw, Bouwe Bolger, "Second harmonic generation of diode laser radiation in KNbO3," Proc. SPIE 1362,

Physical Concepts of Materials for Novel Optoelectronic Device Applications II: Device Physics and Applications, (1 February 1991); doi: $10.1117 / 12.24571$

SPIE Event: Physical Concepts of Materials for Novel Optoelectronic Device Applications, 1990, Aachen, Germany 


\title{
Second harmonic generation of diode laser radiation in $\mathrm{KNbO}_{3}$
}

\author{
N.F. van Hulst, G.J.T. Heesink, H. de Leeuw, B. Bölger \\ Opto-electronics \\ Faculty of Applied Physics \\ University of Twente \\ P.0.Box 217, 7500 AE Enschede, The Netherlands
}

\begin{abstract}
Both CW and pulsed diode lasers covering the wavelength region 855-912 $\mathrm{nm}$ have been investigated for the frequency doubling in $\mathrm{KNbO}_{3}$. The influence of the spectral distribution, mode character and the optical geometry of the laser beam have been investigated for several AlGaAs diode lasers. Second harmonic radiation with maximum efficiency of typically $1 \%$ has been detected both by temperature tuning ( $\mathrm{T}=10-130^{\circ} \mathrm{C}$, noncritical phase matching) and by angle tuning. Results clearly demonstrate the critical aspects of the laser mode spectrum and the beam geometry as to the phase matching criterion in frequency doubling.
\end{abstract}

\section{Introduction}

Compared to other laser systems diode lasers are small, inexpensive and efficient. Therefore they are of great commercial interest. With present available III-V semiconductor materials the emission wavelength of diode lasers is limited to $\sim 633 \mathrm{~nm}$ at room temperature $(\sim 550 \mathrm{~nm}$ at $150 \mathrm{~K})$. Considering the previously mentioned advantageous properties of diode lasers it is obvious that the production of a diode laser emitting in the wavelength region below $633 \mathrm{~nm}$ is desirable (diffraction limit, spectroscopic interest). Radiation in the visible region from diode lasers has been obtained indirectly by frequency doubling of diode laser pumped solid state lasers ( $140 \mathrm{~mW}$ frequency doubled Nd:YAG at $532 \mathrm{~nm}$ is already commercially available [1]). Another approach is the direct frequency doubling of diode lasers. At this end several problems arise as there are, the relative low power of the diode laser and therefore a low frequency doubling efficiency, the wavelength stability (temperature, laser current) and the beam geometry of the diode laser. Thus for the frequency doubling of diode lasers a frequency doubling material with large nonlinear coefficients and noncritical phase match (NCPM) possibility is required. At present $\mathrm{KNbO}_{3}$ is the only material known to posses both qualities. The intention of this paper is to give a review of all parameters of interest considering second harmonic generation ( $\mathrm{SHG}$ ) of AlGaAs diode laser radiation in $\mathrm{KNbO}_{3}$. We have examined SHG of four different types of diode lasers both $\mathrm{CW}$ and pulsed, single and multi mode. The wavelength region covered was $855-912 \mathrm{~nm}$. 


\section{Theory}

\subsection{General theory}

In case of optimum focusation and noncritical phase matching in a crystal of length $L$, nonlinear coefficient $d_{1 j k}$ and refractive index $n$ it is found for the efficiency $\eta$ of the power conversion from fundamental $P(\omega)$ into second harmonic (SH) radiation $P(2 \omega)[2,3]$,

$$
\begin{aligned}
& \eta=\frac{P(2 \omega)}{P(\omega)}=\frac{2}{\pi c}\left(\frac{\mu_{0}}{\varepsilon_{0}}\right)^{\frac{3}{2}} \frac{\omega^{3} d_{1 j k}^{2} L}{n(2 \omega) n(\omega)} P(\omega) \operatorname{sinc}^{2}(\Delta k L / 2) \\
& \text { with, } \Delta k=2 k(\omega)-k(2 \omega)
\end{aligned}
$$

The SH power is effectively generated over the coherence length $l_{c}$, with $l_{c}=2 \pi / \Delta k$. In case $l_{c} \ll L$ Makerfringes [4] are observed when varying the argument of the sincfunction. In case $l_{c} \gg \mathrm{L}$ so called phase matching occurs and $\mathrm{SH}$ radiation is generated over the full length of the crystal. In the latter case birefringence may be used to compensate for the dispersion of the crystal.

Phase matching (PM) only takes place within a limited interval of wavelengths and propagation directions. For this a suitable criterion is [3],

$$
\operatorname{sinc}^{2}(\Delta k(\lambda) \cdot L / 2) \geq 1 / 2 \quad \Rightarrow \quad \Delta k \cdot L / 2 \leq 1.39
$$

It then follows for the acceptance bandwidth,

$$
\delta \lambda=\frac{1.39 \cdot \lambda}{2 \pi \cdot L}\left(\frac{\partial n(\omega)}{\partial \lambda}-\frac{1}{2} \frac{\partial n(2 \omega)}{\partial \lambda}\right)^{-1}
$$

and for the total angle of acceptance,

$$
\delta \vartheta=\frac{1.39 \cdot \lambda}{2 \pi \cdot L}\left(\frac{\partial \mathrm{n}(\omega)}{\partial \vartheta}-\frac{\partial \mathrm{n}(2 \omega)}{\partial \vartheta}\right)^{-1}
$$

\subsection{Potassium niobate $-\mathrm{KNbO}_{3}$}

Potassium niobate is a perovskite type of ferroelectric. Within the temperature range $-40{ }^{\circ} \mathrm{C}$ to $225^{\circ} \mathrm{C}$ the crystal is orthorhombic, point group symmetry mm2 $\left(\mathrm{C}_{2 \mathrm{v}}\right)$ and thus optically bi-axial. The $\mathrm{KNbO}_{3}$ crystal is ferroelectric to $435^{\circ} \mathrm{C}$ it contains numerous spontaneously polarized domains. By poling the crystal it is possible to obtain a single domain crystal [5].

Because of the crystallographic structure of $\mathrm{KNbO}_{3}$ the nonlinear polarization 
reduces to $[2]$,

$$
\left(\begin{array}{l}
P_{a} \\
P_{b} \\
P_{c}
\end{array}\right)=\left(\begin{array}{cccccc}
0 & 0 & 0 & 0 & d_{15} & 0 \\
0 & 0 & 0 & d_{24} & 0 & 0 \\
d_{31} & d_{32} & d_{33} & 0 & 0 & 0
\end{array}\right)\left(\begin{array}{c}
E_{a}^{2} \\
E_{b}^{2} \\
E_{c}^{2} \\
2 E_{b} E_{c} \\
2 E_{a} E_{c} \\
2 E_{a} E_{b}
\end{array}\right)
$$

The axes $a, b$ and $c$ are the principal dielectric axes. They are directly related to the crystallographic axes $x, y$ and $z$ via an orthogonal transformation.

Type I NCPM is considered. The propagation direction of the fundamental beam is along one of the optic axes. The fundamental and harmonic wave are then polarized along the other two optical axes. Considering (5) it can be seen that only two possible situations for type I NCPM remain using the $d_{31}$ or the $d_{32}$ nonlinear coefficient. For the $d_{15}$ and the $d_{24}$ nonlinear coefficients only type II PM is possible (in the far infra-red). For the $d_{33}$ nonlinear coefficient no PM can be achieved because the birefringence can not be used to compensate for the dispersion.
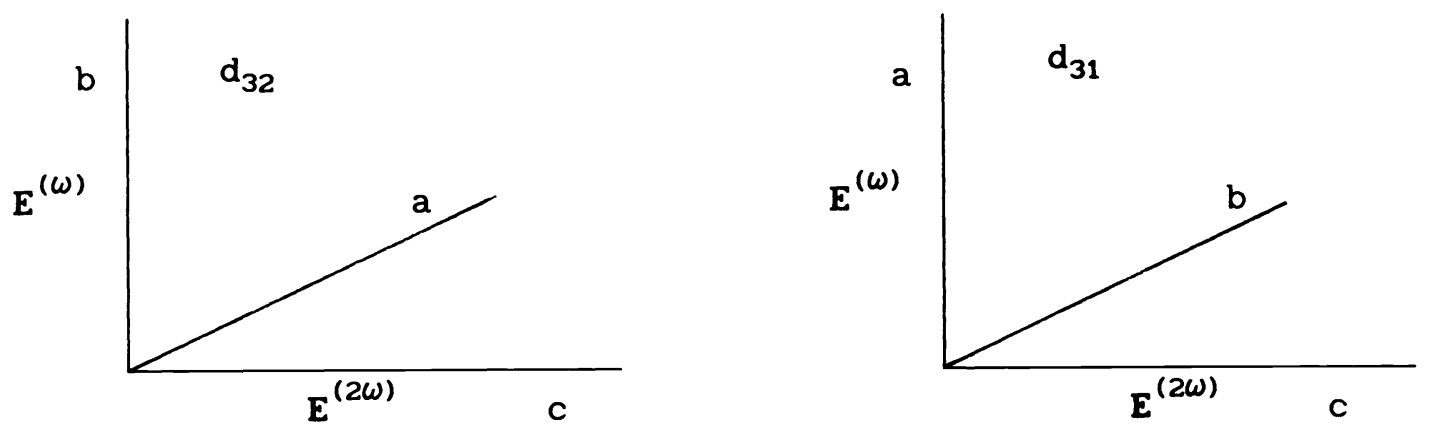

Figure 1: Possible situations for type I NCPM.

The Sellmeier coefficients which describe the refractive indices $n_{a}(\lambda), n_{b}(\lambda)$ and $n_{c}(\lambda)$ have been determined by Wiesendanger [5]. For $T=25^{\circ} \mathrm{C}$ NCPM can be achieved at,

$$
\begin{aligned}
& d_{31} \rightarrow n_{a}\left(\lambda_{f}\right)=n_{c}\left(\lambda_{f} / 2\right) \Rightarrow \lambda_{f}=947 \mathrm{~nm} \\
& d_{32} \rightarrow n_{b}\left(\lambda_{f}\right)=n_{c}\left(\lambda_{f} / 2\right) \Rightarrow \lambda_{f}=860 \mathrm{~nm}
\end{aligned}
$$

If the fundamental beam is in the ac-plane with the polarization along the b-axis critical type I PM is possible. The PM angle as a function of the wavelength is given by,

$$
\sin ^{2}\left(\vartheta_{\mathrm{pm}}(\lambda)\right)=\frac{\mathrm{n}_{\mathrm{b}}^{-2}(\lambda)-\mathrm{n}_{\mathrm{c}}^{-2}(\lambda / 2)}{\mathrm{n}_{\mathrm{a}}^{-2}(\lambda / 2)-\mathrm{n}_{\mathrm{c}}^{-2}(\lambda / 2)}
$$


And for propagation in the bc-plane,

$$
\sin ^{2}\left(\vartheta_{\mathrm{pm}}(\lambda)\right)=\frac{\mathrm{n}_{\mathrm{a}}^{-2}(\lambda)-\mathrm{n}_{\mathrm{c}}^{-2}(\lambda / 2)}{\mathrm{n}_{\mathrm{b}}^{-2}(\lambda / 2)-\mathrm{n}_{\mathrm{c}}^{-2}(\lambda / 2)}
$$

PM angles as a function of the wavelength of the fundamental beam are shown in figure 2.

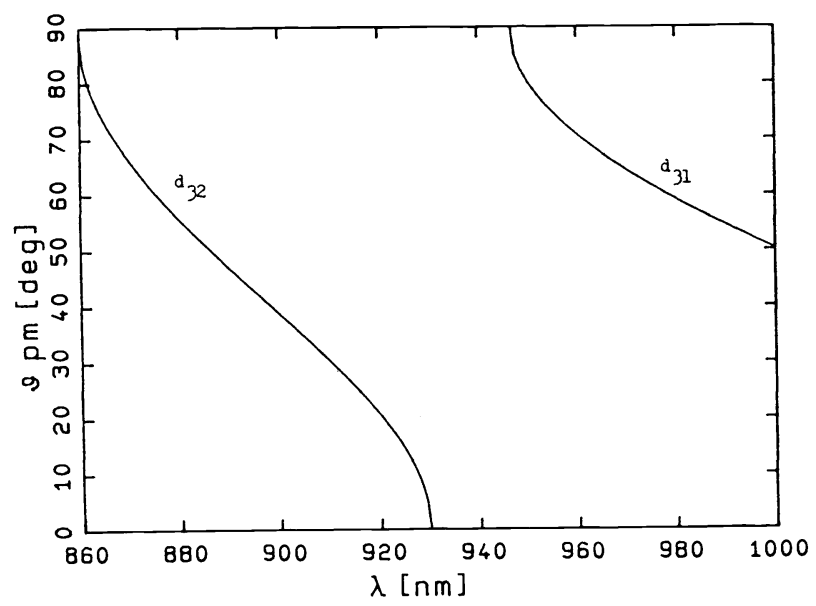

Figure 2: Propagation direction for $P M$ as a function of the fundamental wavelength.

We have calculated the acceptance bandwidth $\Delta \lambda$, the angle of acceptance $\Delta \vartheta$, the PM angle $\vartheta_{\mathrm{pm}}$ as a function of the crystal temperature $T$, the temperature $\mathrm{T}_{\mathrm{ncpm}}$ at which NCPM is possible and the SH power as a function of the angle of incidence and as a function of the crystal temperature.

Thus results from measurements are compared to theoretical expectations.

\section{Experiments}

\subsection{Diode laser characterization}

Frequency doubling of four different types of commercially available diode lasers has been investigated:
STC LT $40-82$
A highly single mode CW laser at $\lambda=855 \mathrm{~nm}$
Philips CQL63
A multi mode CW laser centered at $\lambda=870 \mathrm{~nm}$
RCA SG2012
A pulsed laser at $\lambda=908 \mathrm{~nm}$
STC LJ30
A pulsed laser with low threshold current at $\lambda=862 \mathrm{~nm}$

As the spectral distribution and output power of these lasers are essential in the understanding of their SHG behavior we have measured the absolute power and the laser spectra (using a Bentham M300 HR monochromator, $0.05 \mathrm{~nm}$ resolution) as a function of the laser current and temperature. The measured spectra are shown in the figures $3 a, b, c$ and $d$. The main laser characteristics are summarized in table 1. 

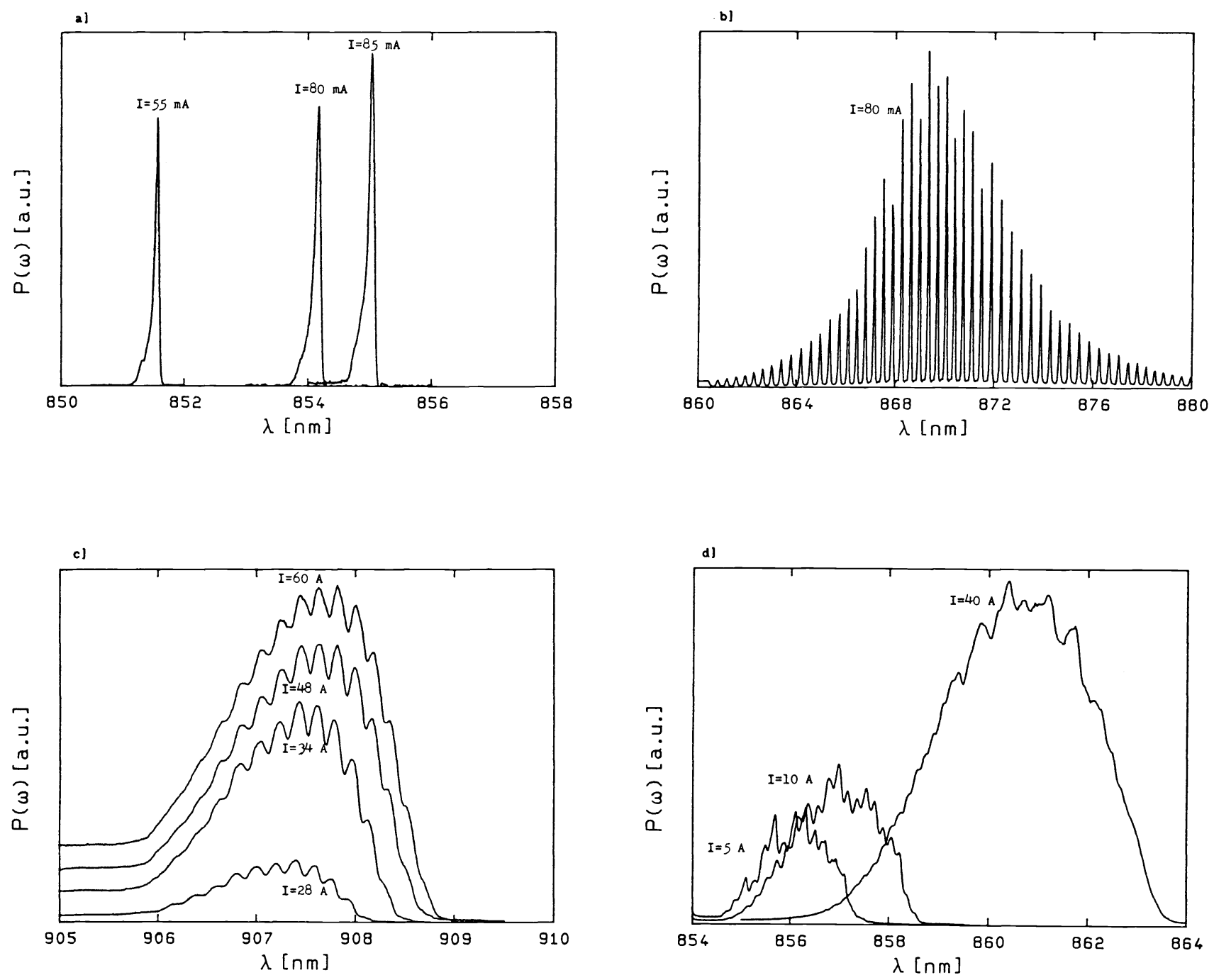

Figure 3 a: The spectrum of the STC LT 40-82 diode laser.

b: The spectrum of the Philips CQL63 diode laser.

c: The spectrum of the RCA SG2012 diode laser.

d: The spectrum of the STC LJ3O diode laser.

Table 1: Summary of the results of the diode laser characterization.

\begin{tabular}{|l|l|l|l|l|c|}
\hline & type & $P[W]$ & $\lambda_{c}[\mathrm{~nm}]$ & $I_{t h}[A]$ & $\Delta \lambda[\mathrm{nm}]$ \\
\cline { 2 - 6 } STC LT 40-82 & CW & $30.10^{-3}$ & 855 & $40.10^{-3}$ & $\sim 0.11$ \\
Philips CQL63 & CW & $5.10^{-3}$ & 870 & $64.10^{-3}$ & 6 \\
RCA SG2012 & pulse & 20 & 908 & 22 & 2 \\
STC LJ30 & pulse & 30 & 862 & 2 & 4 \\
\hline
\end{tabular}

1: $T_{\text {pulse }}=50 \mathrm{~ns}$, Rep. rate $10 \mathrm{kHz}$. 
AlGaAs has large nonlinear coefficients therefore $\mathrm{SH}$ radiation is generated in the laser itself. Only a small fraction of this radiation generated near the output facet within the absorption length is emitted. For two diode lasers the optical power of this radiation was measured, STC LT 40-82 $\rightarrow P_{1} \cong 10 \mathrm{pW}$, RCA SG2012 $\rightarrow$ $P_{2} \cong 700 \mathrm{pW}$.

Regarding the optical power of these lasers (table 1 ) $P_{2} / P_{1} \approx 10^{-6}$ could be expected. The deviation from this value is due to the fact that the active lasing area of this CW laser is much smaller than that of the pulsed laser. Therefore the power density in the pulsed laser is less than in the CW laser.

The power levels measured are negligible compared to the actual SH power as generated in the potassium niobate crystal.

\subsection{Potassium niobate properties}

Two $\mathrm{KNbO}_{3}$ crystals of length $5.28 \mathrm{~mm}$ and $4.20 \mathrm{~mm}$ have been obtained from Sandoz A.G.. The external crystal surfaces are perpendicular to the optical axes. Surfaces perpendicular to the a-axis are polished which allows use of the nonlinear coefficient $d_{32}$ for type I NCPM.

The transmission threshold was measured to be at $400 \mathrm{~nm}$ which is in agreement with the literature value.

The angle as a function of the fundamental wavelength (figure 4) and the fundamental wavelength for NCPM as a function of the crystal temperature (figure 5 ) were measured using a Nd:YAG (Spectra Physics DCR 11 3G) pumped dye laser (Spectra Physics PDL2 with laser dye LDS 867).

Results are in good agreement with theory (figure 2) and with previous results $[3,6]$.

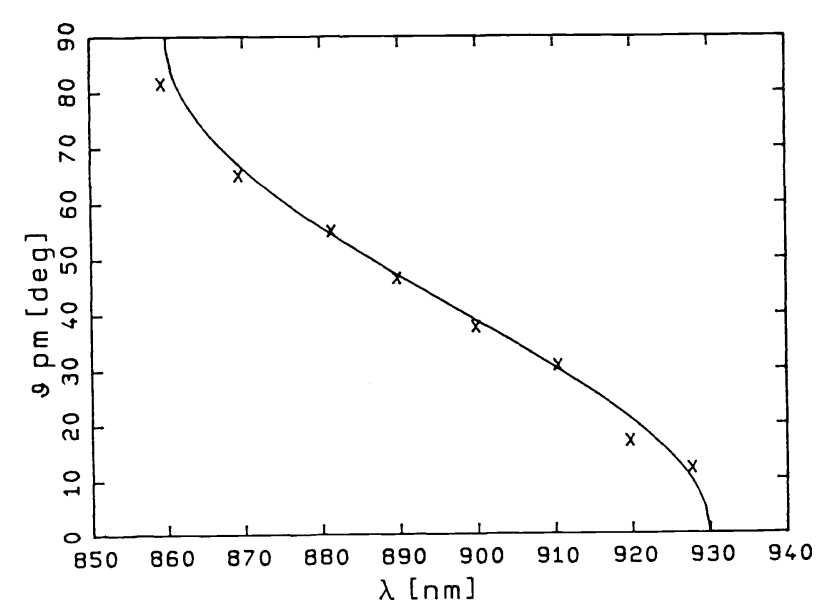

Figure 4: $P M$ angle $\vartheta_{\mathrm{pm}}$ as a function of the fundamental wavelength $\lambda$.

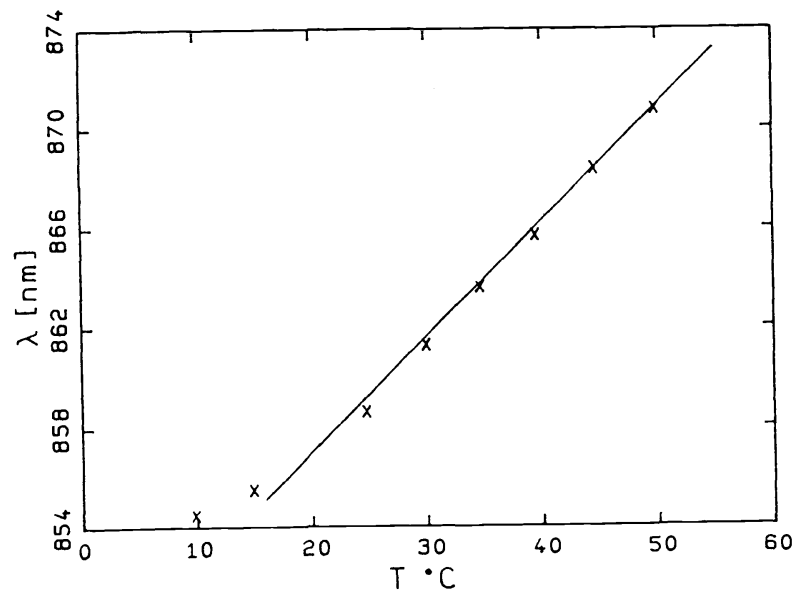

Figure 5: Fundamental wavelength for NCPM as a function of the crystal temperature. 


\subsection{Experimental set-up}

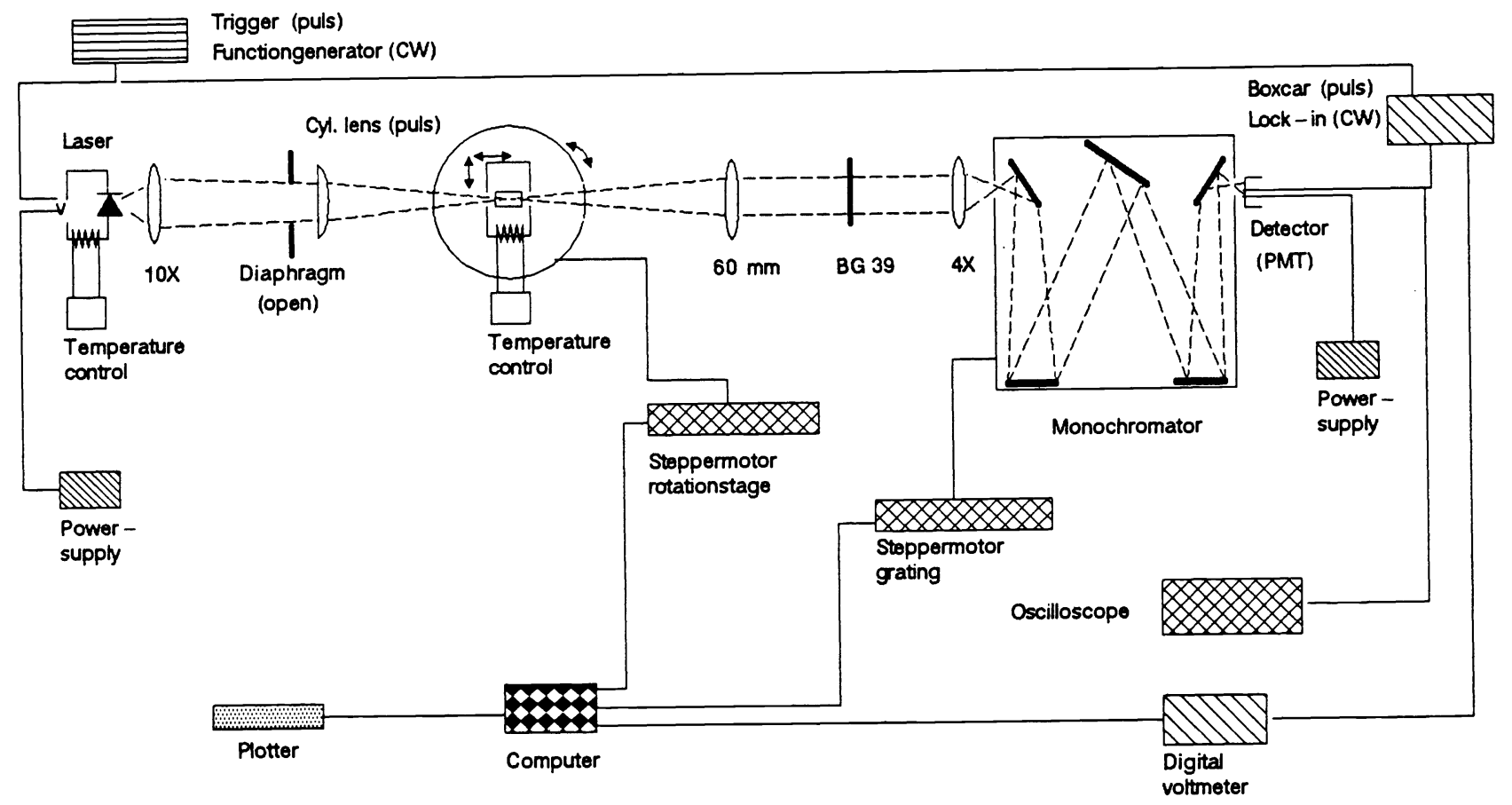

Figure 6: Experimental set-up for the detection of $\mathrm{SHG}$ in $\mathrm{KNbO}_{3}$ from diode laser radiation.

Directly behind the laser an objective with a large NA was positioned to collect as much of the fundamental radiation as possible. In case of the pulsed diode lasers a cylindrical lens was used to compensate for the extreme ellipticity of the beam. The $\mathrm{KNbO}_{3}$ crystal was positioned on a computer controlled rotation stage $\left(\Delta \vartheta=0.001^{\circ}\right)$ in combination with two translation stages which are used for optimum positioning of the laser beam in the crystal. For the temperature tuning of the crystal two different ovens were used one for the temperature range $\mathrm{T}=-10 \rightarrow 50{ }^{\circ} \mathrm{C}$ the other for the range $\mathrm{T}=20 \rightarrow 135^{\circ} \mathrm{C}$. The latter oven is shielded by two $\mathrm{BK} 7$ windows to prevent unwanted cooling of the crystal by draft.

A monochromator (Bentham) was used in combination with a photo multiplier tube (PMT, Hamamatsu R 1463-01) to measure the SH spectrum. To achieve optimum illuminating of the monochromator grating and therefore optimum resolution an objective with the same NA (0.12) as the monochromator was positioned before the monochromator. Measurements on the SHG in $\mathrm{KNbO}_{3}$ were performed under the condition of maximum achievable power except for some measurements on the detection of Makerfringes which demand a beam of small angular spread for which a diaphragm was used. Positions and focal lengths of the lenses used were determined experimentally. 


\section{Results}

The theoretically expected PM conditions for the several lasers are given in table 2 .

Table 2: Theoretical expectations for some parameters of interest considering the frequency doubling of diode lasers in $\mathrm{KNbO}_{3}$.

\begin{tabular}{|l|l|c|c|c|c|c|}
\hline & $\lambda_{c}[\mathrm{~nm}]$ & $\begin{array}{l}\vartheta_{\mathrm{pm}, 25^{\circ} \mathrm{C}} \\
{[\mathrm{deg}]}\end{array}$ & $T_{\mathrm{ncpm}}\left[{ }^{\circ} \mathrm{c}\right]$ & $\begin{array}{c}\Delta \vartheta_{\mathrm{ncpm}} \\
{[\mathrm{deg}]}\end{array}$ & $\begin{array}{l}\Delta \lambda_{\mathrm{ncpm}} \\
{[\mathrm{nm}]}\end{array}$ & $\begin{array}{c}\mathrm{L}[\mathrm{mm}] \\
\mathrm{KNbO}_{3}\end{array}$ \\
\hline STC LT 40-82 & 855 & -- & 14 & 2.7 & 0.085 & 5.28 \\
Philips CQL63 & 870 & 24 & 45 & 3.0 & 0.091 & 5.28 \\
RCA SG2012 & 908 & 58 & 125 & 3.3 & 0.150 & 4.20 \\
STC LJ30 & 862 & 10 & 30 & 2.8 & 0.082 & 5.28 \\
\hline
\end{tabular}

\subsection{The CW diode laser STC LT 40-82}

The temperature of the laser was raised to $31{ }^{\circ} \mathrm{C}$ thus shifting the central wavelength to $\lambda_{c}=856.5 \mathrm{~nm}$. Now $N C P M$ at $13.5{ }^{\circ} \mathrm{C}$ is possible at this temperature no condensation appeared on the crystal.

The SH spectrum is shown in figure 7.

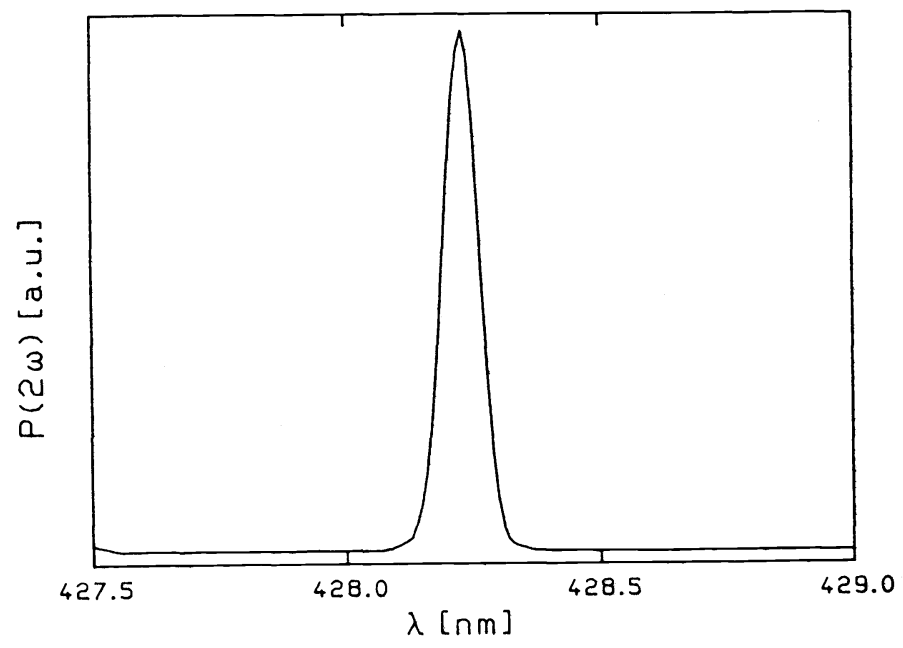

Figure 7: Spectrum of the frequency doubled radiation from the STC LT 40-82 laser in $\mathrm{KNbO}_{3}(5.28 \mathrm{~mm})$.

From figure 7 it can be seen that the acceptance bandwidth $\Delta \lambda \simeq 0.09 \mathrm{~nm}$ which is in reasonable agreement with the theoretically obtained value (table 2 ). The linewidth of the fundamental mode is. within the resolution of the monochromator.

The SH signal as a function of the angle of incidence was measured for $\mathrm{T}=13.5{ }^{\circ} \mathrm{C}$ (NCPM, figure $8 \mathrm{a}$ ) and for $T=13^{\circ} \mathrm{C}$ (critical PM, figure $8 \mathrm{~b}$ ). 
For the situation of NCPM it can be seen that the angle of acceptance is $\Delta \theta \simeq 2.7^{\circ}$ this again is consistent with the calculated value. The beam divergence was measured to be $2.7^{\circ}$ therefore the whole input beam is just within the angle of acceptance. As could be expected in case of critical PM the angle of acceptance is much smaller.

Makerfringes were detected for propagation directions close to the PM angle the (figure 9). Experimental and theoretical data are in good agreement.
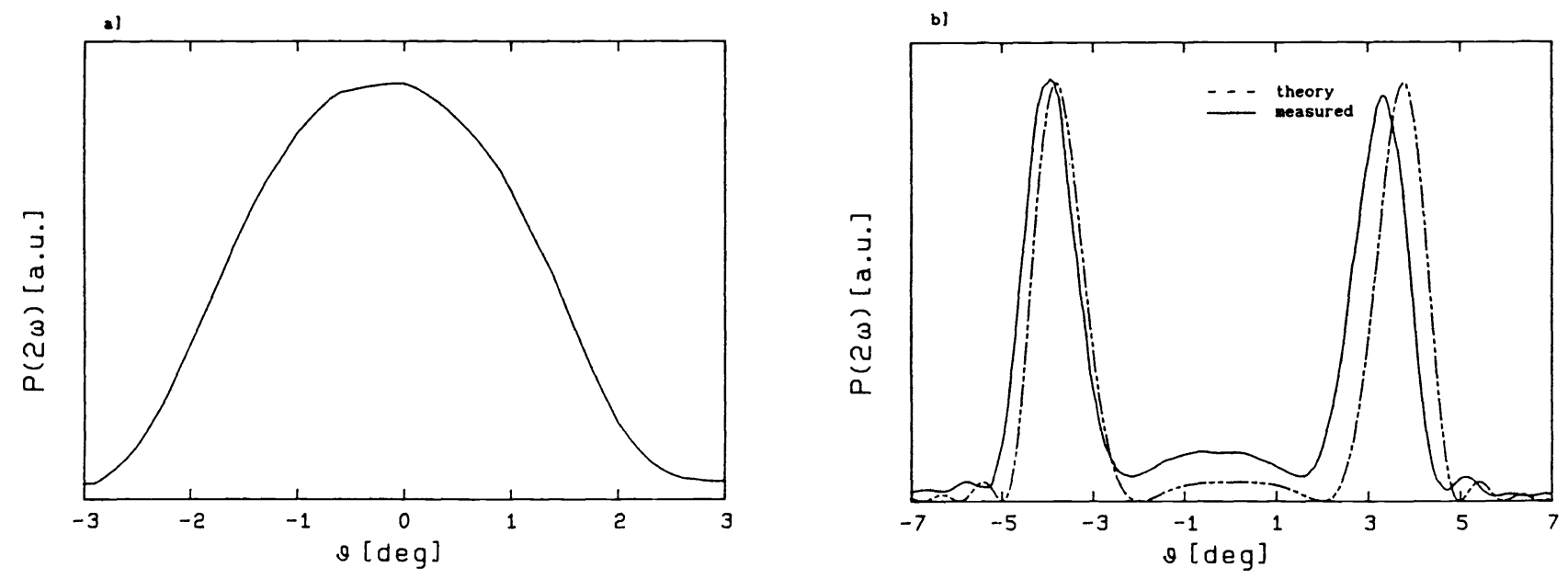

Figure 8: $S H$ power around the $P M$ angle

a: $\operatorname{NCPM}(\theta=0)$.

b: Critical PM.

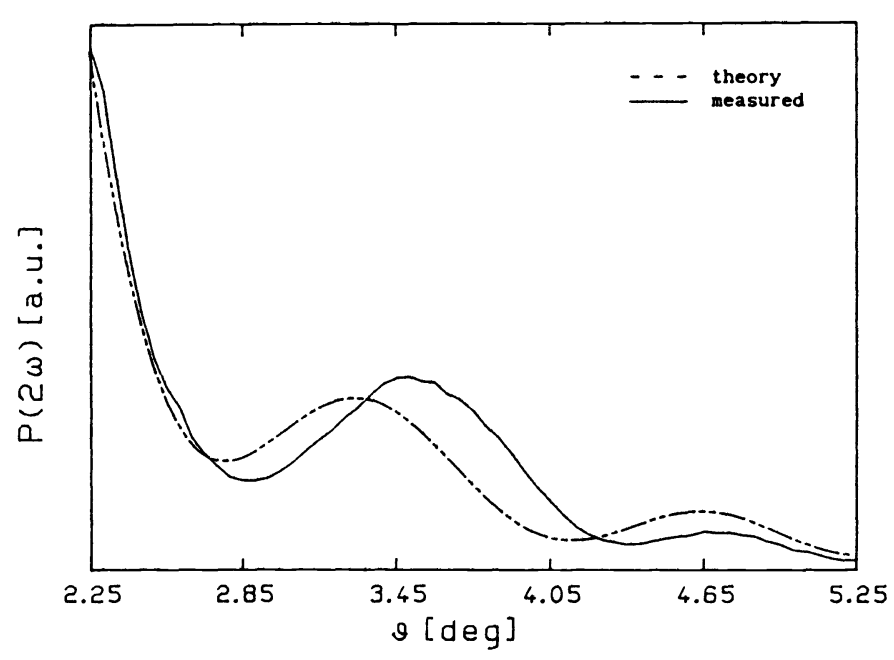

Figure 9: Makerfringes (experimental and theoretical) near the PM angle. 
The SH output in case of NCPM as a function of the crystal temperature is shown in figure 10. The crystal temperature for maximum power is very critical.

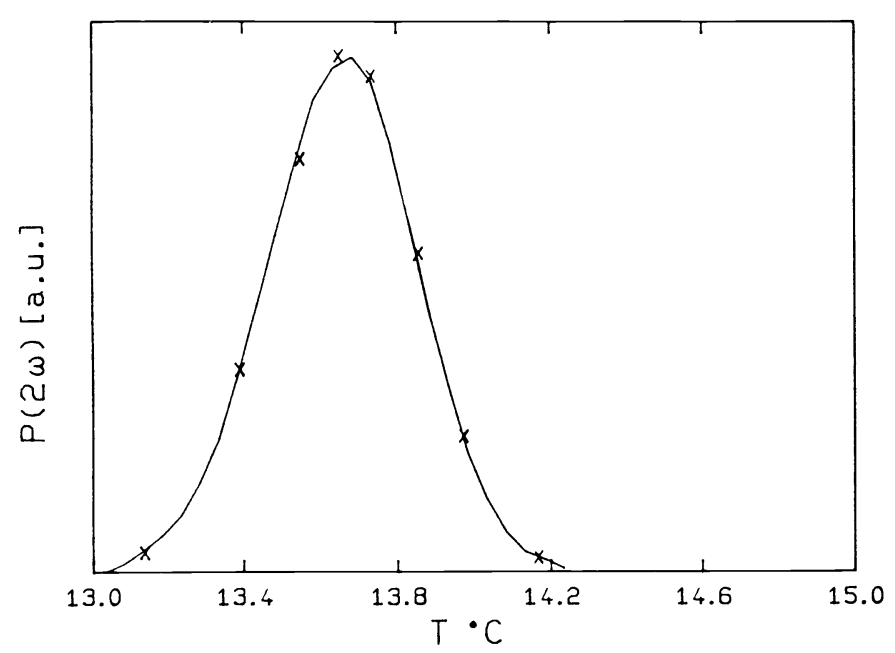

Figure 10: $S H$ power in case of NCPM versus the crystal temperature

The optical power emitted from the laser is $30 \mathrm{~mW}$. From this $25.5 \mathrm{~mW}$ enters the crystal. Because the linewidth of the fundamental mode is within the acceptance bandwidth it may be concluded that $25.5 \mathrm{~mW}$ of fundamental power is within the PM condition. SH power emerging from the crystal was measured to be $P(2 \omega) \simeq 1.7 \mu \mathrm{W}$, in this value losses between crystal and detector were taken into account.

The efficiency is defined as the ratio of the SH power emerging from the crystal and the fundamental power incident on the crystal within the PM condition.

So $\eta=P(2 \omega) / P(\omega) \simeq 7 \cdot 10^{-5}$.

\subsection{The CW diode laser Philips CQL63}

To measure the SH spectrum the aperture of the diaphragm (figure 6) was reduced. Thus the beam incident on the crystal was almost parallel and the SH spectrum is a measure for the acceptance bandwidth. The spectrum for NCPM is shown in figure 11a. The acceptance bandwidth $(\sim 0.09 \mathrm{~nm})$ is in agreement with the calculated value. Measurements with open diaphragm are shown in figure $11 \mathrm{~b}$ and 11c. The acceptance bandwidth is much smaller in case of critical PM compared to the case of NCPM. This can be explained by the large value of $\partial \vartheta_{\mathrm{pm}} / \partial \lambda$ at $\vartheta_{\mathrm{pm}} \cong 90^{\circ}$ (figure 2).

No Makerfringes could be detected. If the diaphragm is opened several modes of the fundamental beam are incident on the crystal. It can be calculated that because of the angular and spectral spread of the fundamental beam the modulation depth of the generated fringes is almost zero. For a diaphragm with reduced aperture the fundamental power (one mode, $0.25 \mathrm{~mW}$ ) incident on the crystal is too low for the detection of $\mathrm{SH}$ radiation at non phase match condition.

The SH signal around the $\mathrm{PM}$ angle was measured at $\mathrm{T}=25^{\circ} \mathrm{C}$ and at $\mathrm{T}=44.5{ }^{\circ} \mathrm{C}$ (figure 12). At the latter temperature $P M$ is achieved with $\Delta \vartheta \simeq 2.8^{\circ}$. For $\mathrm{T}=25^{\circ} \mathrm{C}$ it can be seen $\vartheta_{\mathrm{pm}}\left(\mathrm{T}=25^{\circ} \mathrm{C}\right)=24^{\circ}$ and $\Delta \vartheta\left(\mathrm{T}=25^{\circ} \mathrm{C}\right)=0.2^{\circ}$. The parameters measured are in good agreement with the calculated values. 

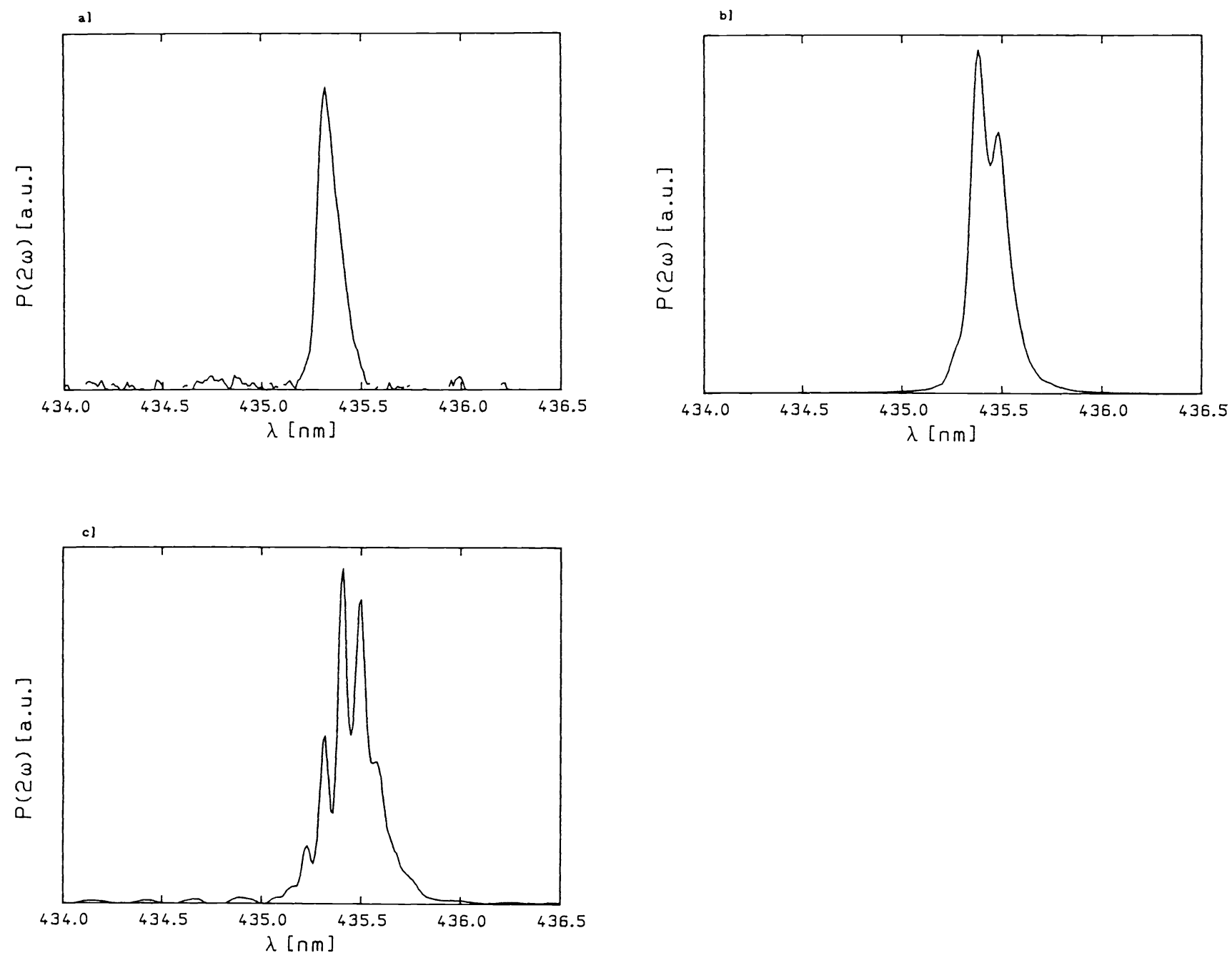

Figure 11: Spectrum of the frequency doubled radiation from the Philips CQL63 laser in $\mathrm{KNbO}_{3}$

a: NCPM, reduced aperture.

b: NCPM, open diaphragm.

c: critical PM, open diaphragm.

In case of NCPM approximately 2 modes of the fundamental beam are within the acceptance bandwidth. This corresponds to a fundamental power $P(\omega)=0.5 \mathrm{~mW}$ (losses between laser and crystal were taken into account). Considering the difference in acceptance bandwidth for the cases of NCPM and critical PM (figure 11b and 11c) possibly for the case of a beam with considerable angular and spectral spread the frequency doubling efficiency needs not to be at a maximum for NCPM.

The angular spread is $3^{\circ}$ and thus almost completely within the angle of acceptance. The SH power at NCPM was measured, $P(2 \omega)=2.3 \mathrm{nW}$.

Thus $\eta=\mathrm{P}(2 \omega) / \mathrm{P}(\omega)=4.5 \cdot 10^{-6}$. 

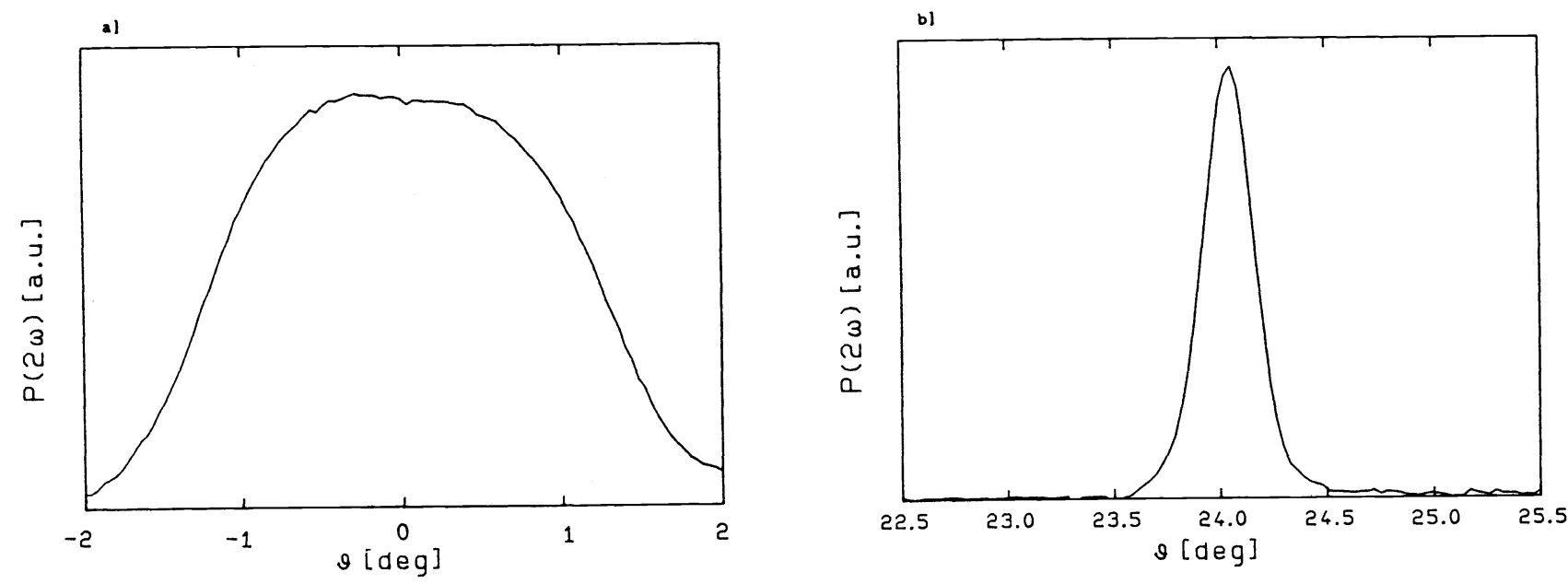

Figure 12: $S H$ power around the PM angle a: NCPM.

b: Critical PM.

\subsection{The RCA SG2012 pulsed diode laser}

To achieve NCPM for the RCA SG2012 diode laser the temperature of the crystal has to be $\mathrm{T}=125{ }^{\circ} \mathrm{C}$. During the heating (and cooling) of the crystal several 'cracks' (inverted ferroelectric domains) appeared inside the crystal. For this reason an other $\mathrm{KNbO}_{3}$ crystal had to be used.

The SH spectrum of the RCA diode laser was measured with open diaphragm (figure 13). In this case the acceptance bandwidth is partially determined by the angular spread of the beam.

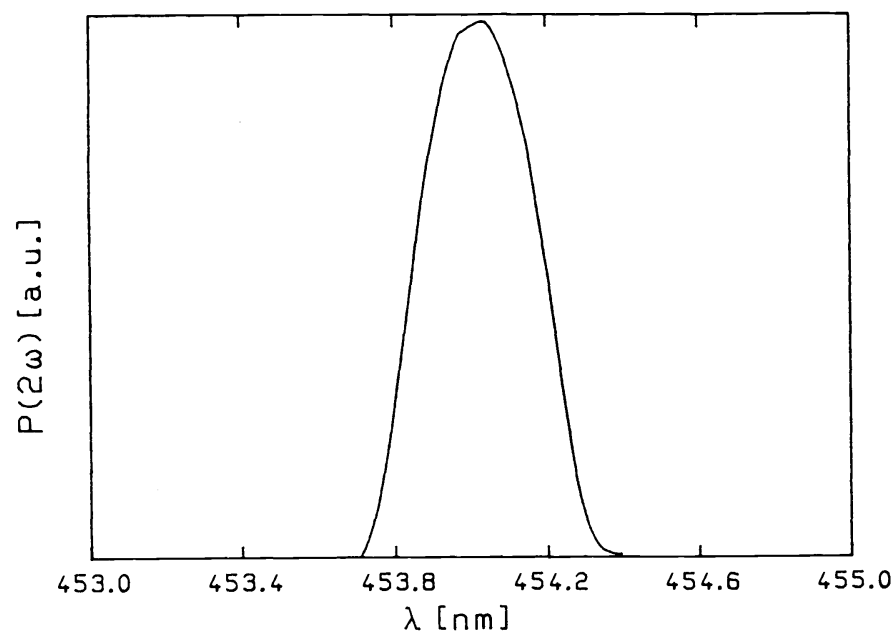

Figure 13: Spectrum of the frequency doubled radiation from the RCA SG2012 laser in $\mathrm{KNbO}_{3}(4.20 \mathrm{~mm})$. 
At temperature $\mathrm{T}=25^{\circ} \mathrm{C}$ Makerfringes were detected near the PM angle (figure 16).

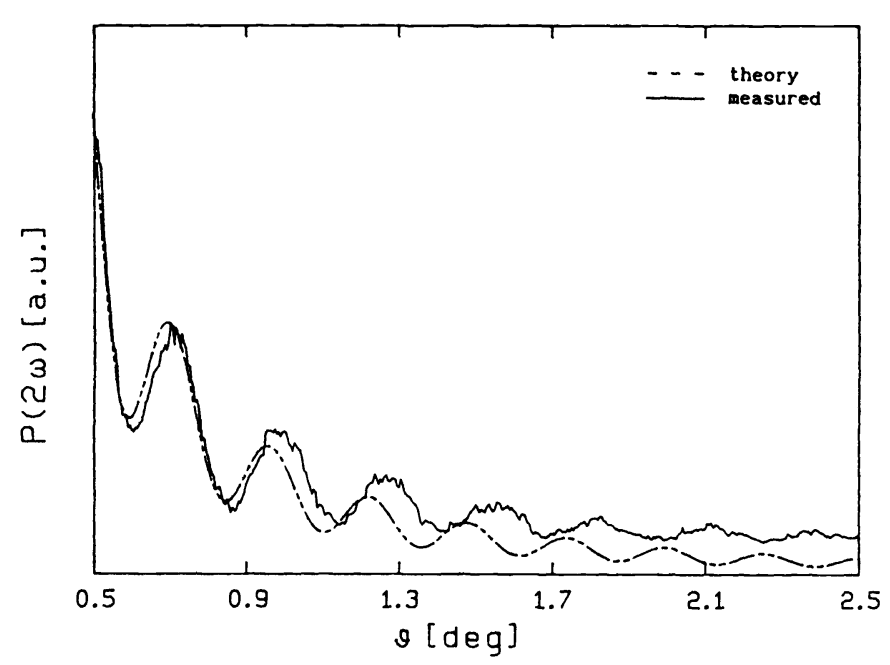

Figure 16: Makerfringes (experimental and theoretical) near the PM angle.

From the measured values of the acceptance bandwidth and the angle of acceptance we conclude that $P(\omega) \simeq 0.6 \mathrm{~W}$ of fundamental power is within the PM condition. The SH power measured was $P(2 \omega) \simeq 0.6 \mathrm{~mW}$.

Thus, $\eta=\mathrm{P}(2 \omega) / \mathrm{P}(\omega) \simeq 10^{-3}$.

\subsection{The STC LJ30 pulsed diode laser}

The SH spectrum was measured with reduced aperture (figure 17a) and with open diaphragm (figure 17b).
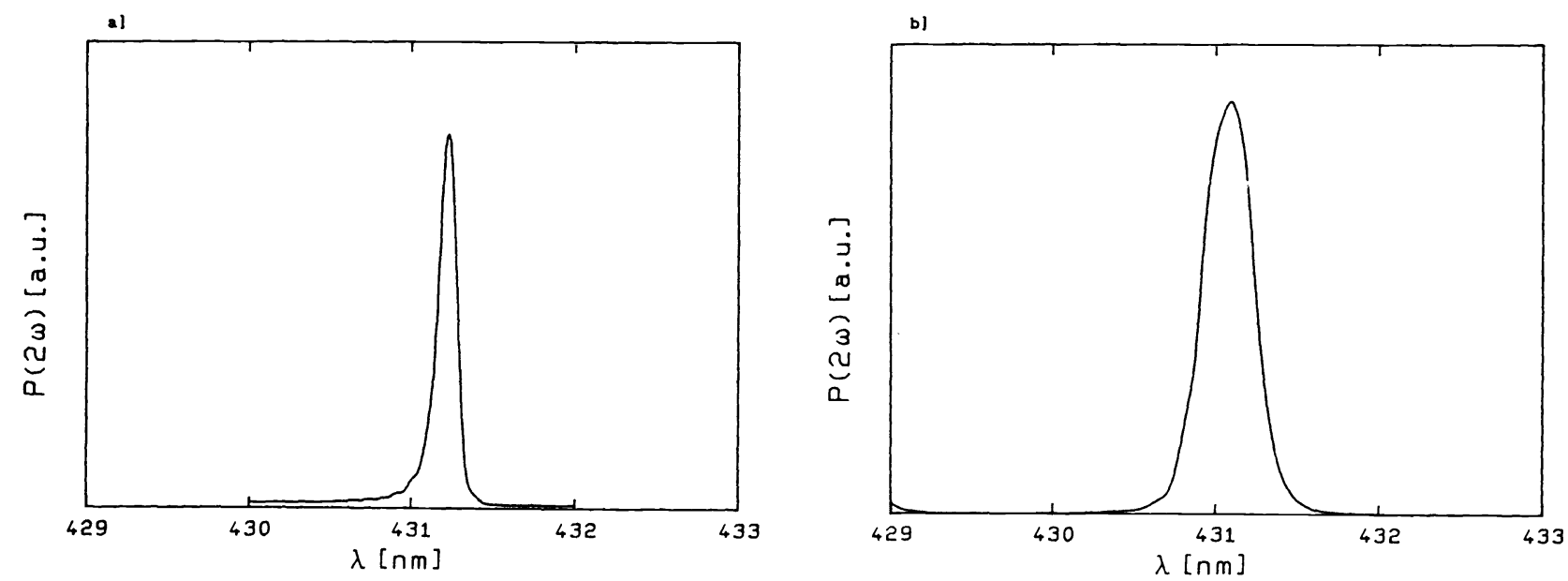

Figure 17: Spectrum of the frequency doubled radiation from the STC LJ3O laser in $\mathrm{KNbO}_{3}(5.28 \mathrm{~mm})$

a: reduced aperture.

b: open diaphragm. 
The SH signal around the $\mathrm{PM}$ angle was measured at $\mathrm{T}=25^{\circ} \mathrm{C}$ and at $\mathrm{T}=125^{\circ} \mathrm{C}$ ( $\mathrm{figure}$ 14). Because of the large angular and spectral spread of the fundamental beam the angle of acceptance measured is much larger $\left(\Delta \vartheta_{\mathrm{ncpm}} \cong 8^{\circ}, \Delta \vartheta_{\mathrm{cpm}} \cong 6^{\circ}\right)$ than the theoretically calculated angle of acceptance. The PM angles measured at both temperatures are in agreement with the calculated values.
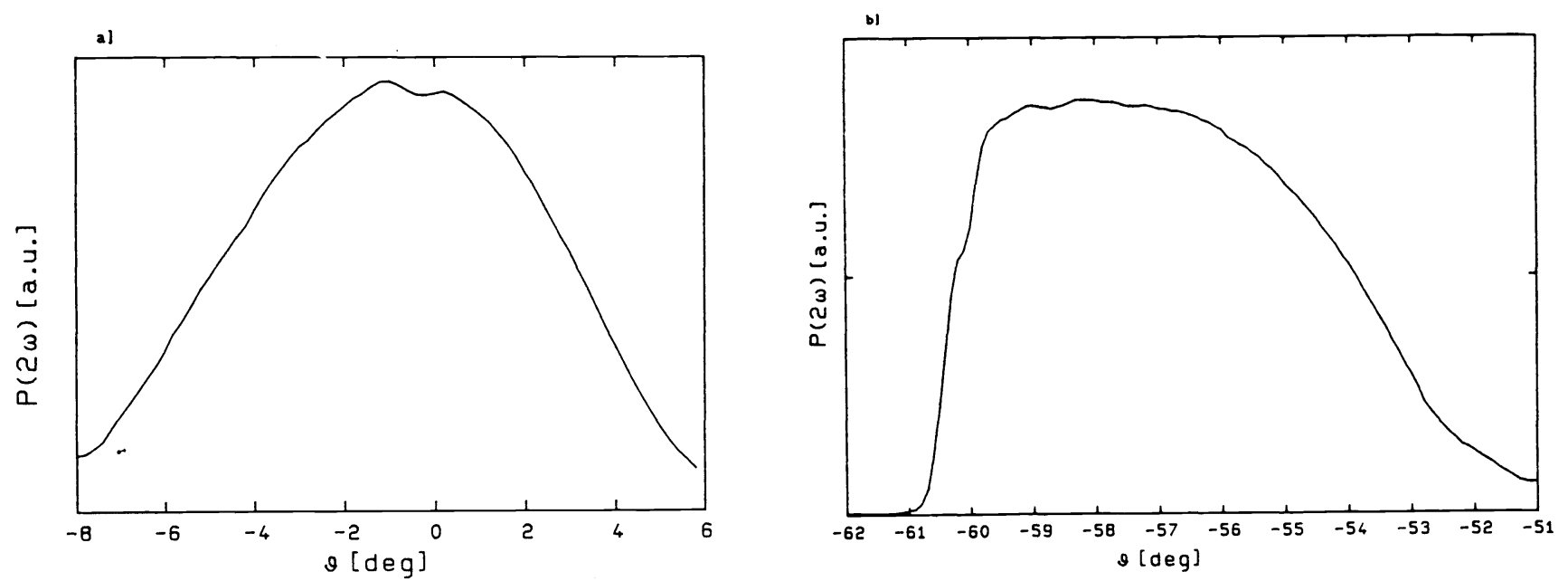

Figure 14: $S H$ power around the PM angle

a: NCPM.

b: Critical PM.

For the several wavelength components of the fundamental beam PM occurs at different angles. A measurement of the SH signal around the PM angle for several wavelengths is shown in figure 15.

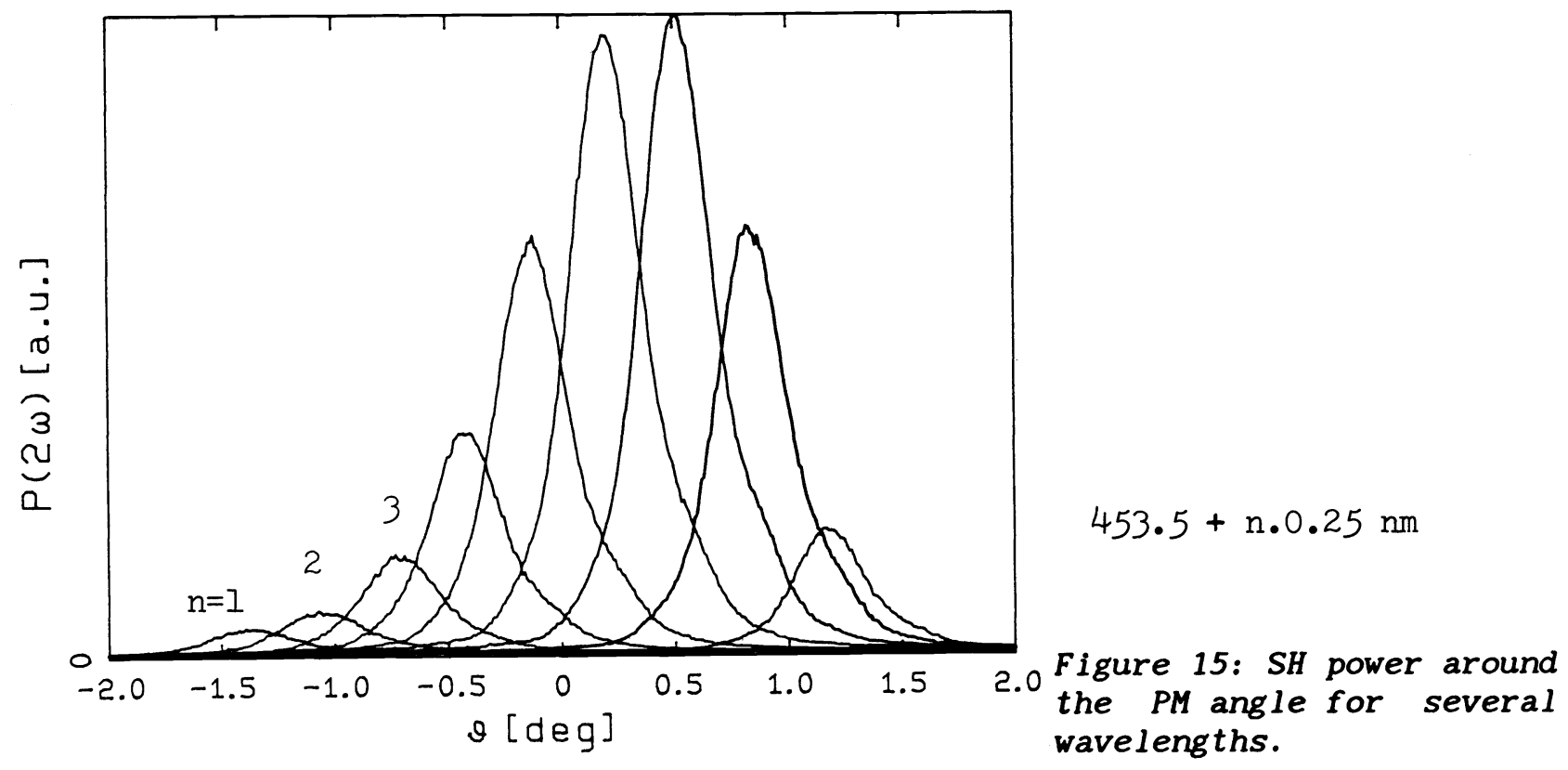


For several temperatures the SH signal was measured at $\lambda=431.6 \mathrm{~nm}$ around the PM angle. The increase in acceptance bandwidth can clearly be seen (figure 18). Results are in agreement with the calculated values.

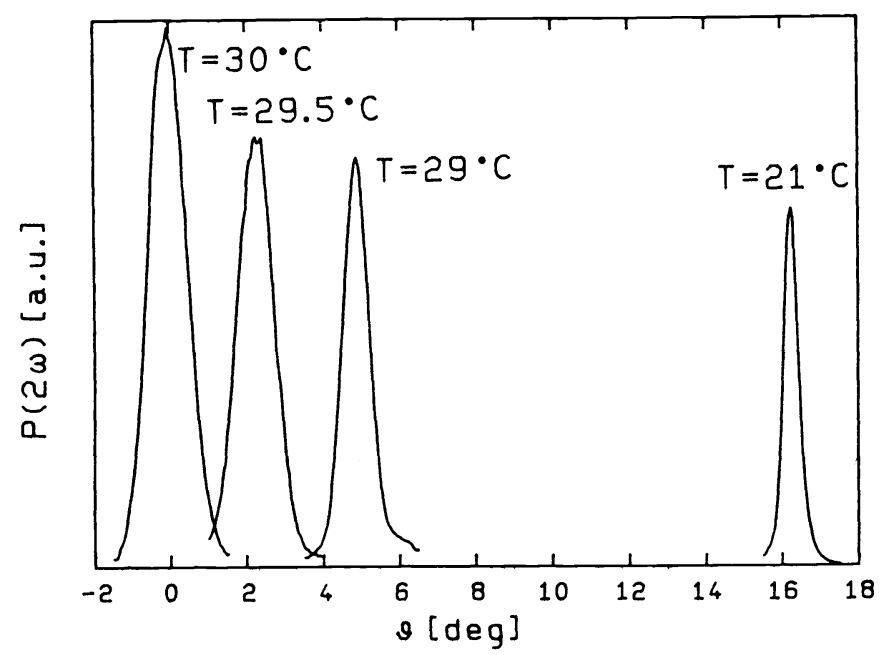

Figure 18: $S H$ power around the $P M$ angle for several temperatures.

From the measured values of the acceptance bandwidth and the angle of acceptance it can be concluded that $P(\omega) \simeq 0.9 \mathrm{~W}$. The SH power measured was $P(2 \omega) \simeq 7 \mathrm{~mW}$.

Thus $\eta=P(2 \omega) / P(\omega) \simeq 8 \cdot 10^{-3}$.

\section{Conclusions and discussion}

For the four different type of diode lasers wavelength, angular and temperature dependent measurements have been performed. Results are in good agreement with expectations from theory.

For the several lasers the efficiency of conversion from fundamental to SH radiation was determined. These values were compared to the theoretically calculated values for the efficiency, $\eta_{t h}$.

Theoretical values of the efficiency $\eta$ were calculated for the case of optimal focusation. Because of the large astigmatism of the laser beam this assumption is probably not realistic and may be the explanation for the difference between measured and calculated values of the efficiency. Previously it has been found by

Table 3: Measured and calculated efficiencies for SHG in $\mathrm{KNbO}_{3}$.

\begin{tabular}{|l|l|l|}
\cline { 2 - 3 } \multicolumn{1}{c|}{} & $\eta_{\text {measured }}$ & $\eta_{\text {theory }}$ \\
\hline STC LT40-82 & $7.0 \cdot 10^{-5}$ & $6.0 \cdot 10^{-4}$ \\
Philips CQL63 & $4.5 \cdot 10^{-6}$ & $1.5 \cdot 10^{-5}$ \\
RCA SG2012 & $1.0 \cdot 10^{-3}$ & $9.5 \cdot 10^{-3}$ \\
STC LJ30 & $8.0 \cdot 10^{-3}$ & $2.9 \cdot 10^{-2}$ \\
\hline
\end{tabular}


P. Günter $[3,6] \quad \eta_{\text {measured }} / \eta_{\text {theory }}=8.1$ which is comparable to our result for the STC LT40-82 and the RCA SG2012 diode lasers. For the Philips CQL63 CW and the STC LJ30 diode lasers the ratio of the measured and the theoretically obtained values for the efficiency was in our case more appropriate.

The highest efficiency was obtained for the STC LJ30 pulsed diode laser, $\eta \simeq 0.8 \%$. The maximum achievable generation of $\mathrm{SH}$ power was most easily obtained for both pulsed lasers because of their broad and continuous spectrum and the high peak power. Therefore the PM condition is less dependent of the angle of incidence (or angular spread) and of the temperature of the crystal. Because of their broad spectrum only a small part of the fundamental radiation from these pulsed lasers is within the PM condition. The repetition frequency of the pulsed lasers was $10 \mathrm{kHz}$ therefore the CW equivalent efficiency is by a factor 2000 less than the efficiency quoted in table 3. Due to these effects the CW equivalent efficiency of the pulsed lasers is less than the efficiency of the CW lasers.

In case of direct frequency doubling of diode laser radiation the efficiency can be enhanced by using more powerful narrow bandwidth lasers and longer $\mathrm{KNbO}_{3}$ crystals. Considering the small acceptance bandwidth $(\partial \vartheta / \partial \lambda$, figure 1$)$ at NCPM related to the considerable angular spread of the laser beam possible enhancement of the SHG efficiency can be obtained by $P M$ at angles somewhat deviating from perpendicular incidence.

To achieve even considerable higher efficiencies intra cavity frequency doubling has been employed [7]. Recently efficiencies up to $17 \%$ have been reported.

\section{Acknowledgements}

We would like to thank F.B. Segerink for his outstanding technical support. This research was supported by funds of the 'Stichting voor Fundamenteel Onderzoek der Materie (FOM)'.

\section{References}

[1] Frequency doubled laser diode pumped Nd:YAG laser: Amoco, Adlas, Spectra Diode Labs.

[2] A. Yariv, Quantum Electronics, John Wiley, New York, 1975.

[3] J.C. Baumert, P. Günter, H. Melchior, 'High efficiency second harmonic generation in $\mathrm{KNbO}_{3}$ crystals', Optics Comm. 48 (3), p.215, 1983.

[4] P.D. Maker, R.W. Terhune, M. Nisenoff and C.M. Savage, Phys. Rev. Lett. 8, p.21, 1962.

[5] E. Wiesendanger, 'Optical properties of $\mathrm{KNbO}_{3}$ ', Ferroelectrics, 1, p.141, 1970.

[6] P. Günter, P.M. Asbeck, S.K. Kurtz, 'Second harmonic generation with $\mathrm{Ga}_{1-x} \mathrm{Al}_{x} \mathrm{As}$ lasers and $\mathrm{KNbO}_{3}$ crystals', Appl. Phys. Lett. 35, p. 461, 1979.

[7] T. Baer, M. Keirstad, Cleo (1990), ThM5. 